\title{
Small bowel adenocarcinoma incarcerated within an inguinal hernia
}

\author{
Hironari Kawai ${ }^{1,2^{*}}$, Koichiro Haruki ${ }^{2}$, Naoki Takada ${ }^{1,2}$, Toshio lino ${ }^{1,2}$, Masahiro Ikegami ${ }^{3}$ and Katsuhiko Yanaga ${ }^{2}$
}

\begin{abstract}
Background: Small bowel adenocarcinomas are rare malignant tumors that account for less than $2 \%$ of gastrointestinal malignancies. In addition, tumor incarceration in an inguinal hernia is also rare entity. We herein report a first case of small bowel adenocarcinoma incarcerated within an inguinal hernia.

Case presentation: A 75-year-old man with asymptomatic anemia (hemoglobin, $8.6 \mathrm{~g} / \mathrm{dl}$ ) had a checkup at our hospital. Colonoscopy revealed bleeding through the ileocecal valve and an annular stricture by a tumor in the ileum. Endoscopic biopsy revealed a well-differentiated adenocarcinoma of the small bowel. Enhanced computed tomography showed a hypervascular solid tumor incarcerated within a right inguinal hernia. With a diagnosis of small bowel adenocarcinoma incarcerated within a right inguinal hernia, the patient underwent elective laparotomy with midline excision. The small bowel tumor, located at $30 \mathrm{~cm}$ from the terminal ileum, was incarcerated within a right inguinal hernia, and the small bowel was adherent to the hernia sac. A 24-cm segment of the distal ileum and regional lymph nodes were resected. The hernia sac was ligated, and the bottom of the hernia sac was resected. The hernia orifice was closed by tissue repair technique via a standard oblique incision in the right inguinal region. Postoperatively, the patient remains well with no evidence of tumor or hernia recurrence as of 1 year after operation.
\end{abstract}

Conclusions: We reported to our knowledge the first case of small bowel adenocarcinoma incarcerated within an inguinal hernia.

Keywords: Small bowel cancer, Small intestinal adenocarcinoma, Incarceration

\section{Background}

Adenocarcinoma of the small bowel is rare and accounts for less than $2 \%$ of gastrointestinal malignancies, which is usually detected by video capsule endoscopy [1]. Tumor incarceration within an inguinal hernia is also rare with an estimated incidence of $<0.5 \%$, which is usually found during emergency operation for the incarcerated hernia. We herein report a first case of small bowel adenocarcinoma incarcerated within an inguinal hernia, which was successfully diagnosed preoperatively by colonoscopy and computed tomography (CT).

\footnotetext{
* Correspondence: h-kawai@jikei.ac.jp

'Department of Surgery, The Saitama Jikei Hospital, 3-208, Ishihara,

Kumagaya, Saitama 360-0816, Japan

${ }^{2}$ Department of Surgery, The Jikei University School of Medicine, 3-25-8,

Nishi-Shimbashi, Minato-ku, Tokyo 105-8461, Japan

Full list of author information is available at the end of the article
}

\section{Case presentation}

A 75-year-old man with asymptomatic anemia (hemoglobin, $8.6 \mathrm{~g} / \mathrm{dl}$ ) had a checkup at our hospital. The patient had no significant past medical history, family history, or predisposing conditions such as familial adenomatous polyposis, hereditary non-polyposis colorectal cancer, Peutz-Jeghers syndrome, and Crohn's disease. The patient was noted to have a non-reducible right inguinoscrotal hernia on examination. The serum carcinoembryonic antigen and carbohydrate 19-9 were within normal range. Colonoscopy revealed bleeding through the ileocecal valve, and therefore, we advanced the scope into the ileum and found an annular stricture by a tumor, for which endoscopic biopsy and clipmarking of the tumor were performed (Fig. 1a). The biopsy revealed well-differentiated adenocarcinoma. Enhanced CT showed a hypervascular solid tumor and a metal clip in the right inguinal hernia (Fig. 1b). With a diagnosis of small bowel adenocarcinoma incarcerated within a right inguinal hernia, the patient underwent 

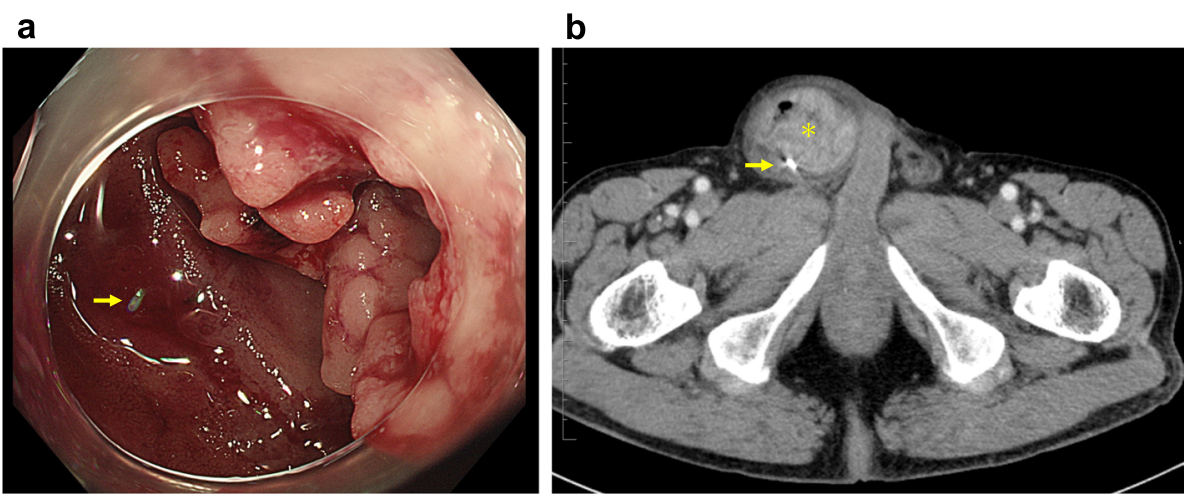

Fig. 1 a Colonoscopy revealed bleeding through the ileocecal valve. Therefore, we advanced the scope into the ileum and found an annular stricture by a tumor, for which endoscopic biopsy and clip-marking (arrow) of the tumor were performed. b Enhanced CT demonstrated a hypervascular solid tumor (asterisk) and a metal clip (arrow) by colonoscopy in the right inguinal hernia

elective laparotomy with midline excision. There was no metastatic lesion including liver metastasis and peritoneal dissemination. The small bowel tumor, located at 30 $\mathrm{cm}$ from the terminal ileum, was incarcerated within a right inguinal hernia, and the small bowel was adherent to the hernia sac although there was no evidence of direct invasion into the hernia sac (Fig. 2a). After adhesiolysis, the tumor and the hernia sac were reduced into the peritoneal cavity (Fig. 2b). A 24-cm segment of the distal ileum and regional lymph nodes were resected, and triangle anastomosis was performed for reconstruction. The reduced hernia sac was ligated, and the bottom of the hernia sac was resected from the peritoneal cavity side (Fig. 2c). Finally, the hernia orifice was closed by tissue repair technique via a standard oblique incision in the right inguinal region. To avoid possible infection, we

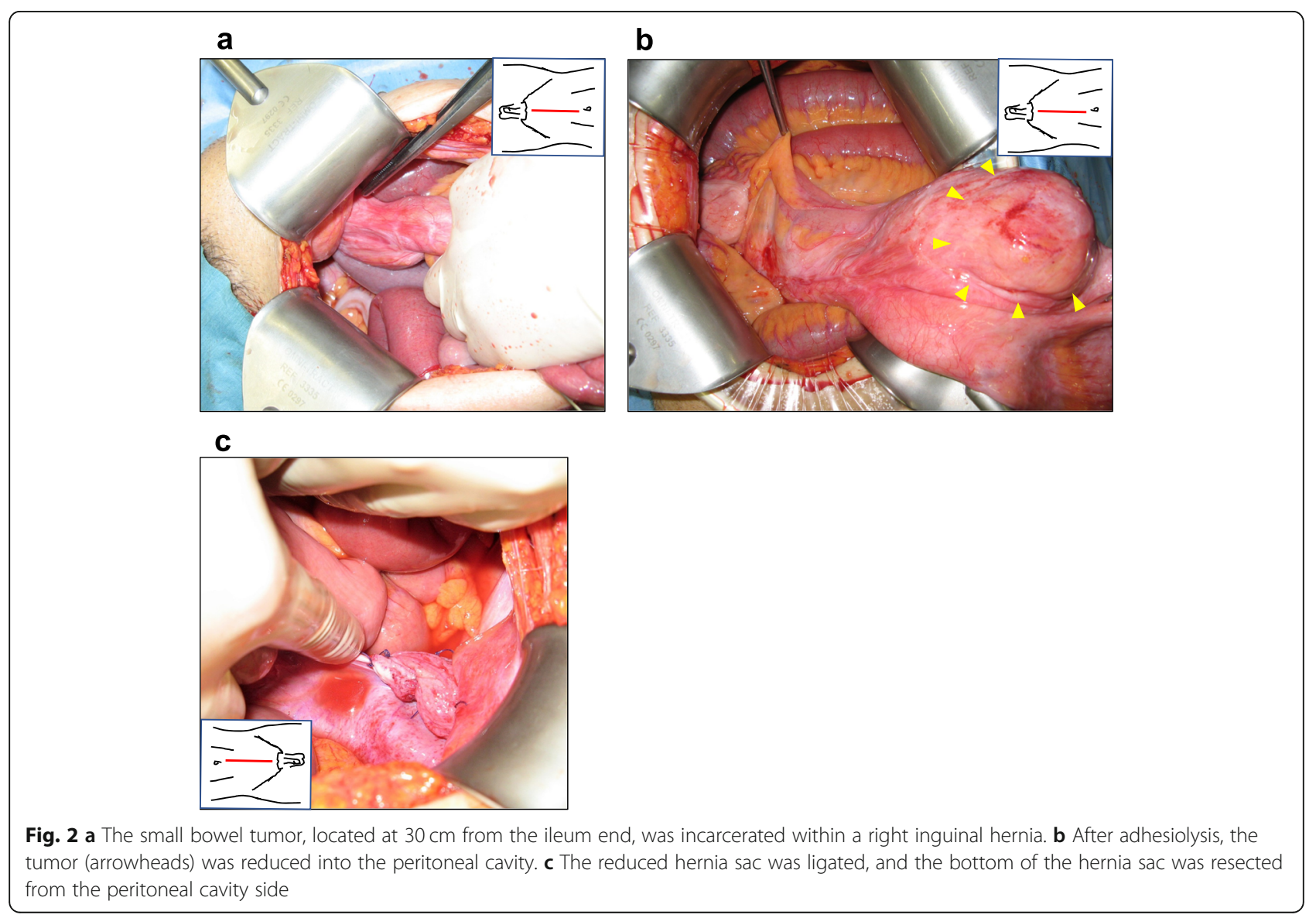


a

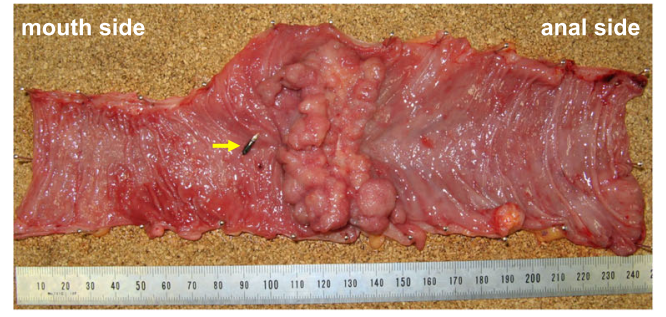

b

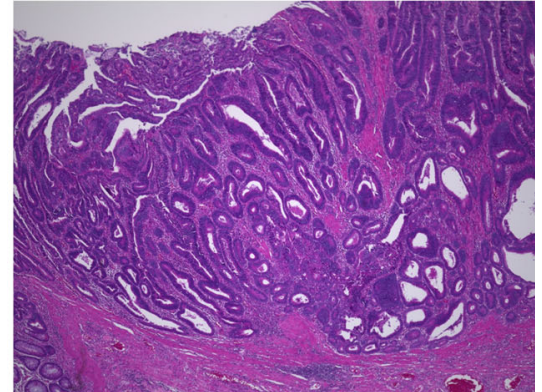

Fig. 3 a The excised specimen revealed a type $V$ (unclassifiable type) tumor with the diameter of $70 \times 40 \mathrm{~mm}$ with nodule-aggregating lesions and a localized ulcer. The marking clip (arrow) was located at $1 \mathrm{~cm}$ distal of the tumor. b Pathological examination revealed well-differentiated adenocarcinoma of the small bowel with no lymph node metastasis

chose a tissue repair technique without a mesh. The excised specimen revealed type $\mathrm{V}$ (unclassifiable type) tumor with the diameter of $70 \times 40 \mathrm{~mm}$ with noduleaggregating lesions and a localized ulcer (Fig. 3a). Pathological examination revealed well-differentiated adenocarcinoma of the small bowel with no lymph node metastasis [Union International Control Cancer classification of malignant tumors 7th edition; pT2, pN0, pM0, stage I, R0] (Fig. 3b). Postoperatively, the patient was discharged on postoperative day 8 without complications. The patient did not receive adjuvant therapy and remains well with no evidence of tumor or hernia recurrence as of 1 year after operation.

\section{Discussion}

This is the first case report of small bowel adenocarcinoma incarcerated within an inguinal hernia in the English literature. Adenocarcinoma of the small bowel is rare and accounts for less than $2 \%$ of gastrointestinal malignancies, and tumor incarceration within an inguinal hernia is also rare with an estimated incidence of $<0.5 \%$; therefore, small bowel adenocarcinoma incarcerated within an inguinal hernia is extremely rare $[1,2]$. Furthermore, the present case has some noteworthy points. First, small bowel adenocarcinoma within an inguinal hernia was successfully diagnosed preoperatively at stage I. Since most small bowel cancers remain asymptomatic until advanced, the majority of patients are diagnosed at advanced stages (32\% stage IV, 27\% stage III, 30\% stage II, and 10\% stage I) [3]. Besides, Kanemura et al. reviewed 31 cases of colon carcinoma incarcerated within an inguinal hernia and reported only a half of the cases were diagnosed preoperatively, while the other half were diagnosed incidentally during emergency operation for incarcerated hernia [4]. Therefore, small bowel adenocarcinoma incarcerated within an inguinal hernia which was preoperatively diagnosed at stage I was rare condition. Second, the tumor was located in the ileum which is a rather rare site for small bowel adenocarcinoma. Although small bowel adenocarcinomas are found throughout the length of the small bowel, more than half (56\%) are located in the duodenum, while tumors of the jejunum and ileum represent $30 \%$ and $20 \%$, respectively [5]. Therefore, tumor location itself was relatively rare. Finally, small bowel adenocarcinoma within an inguinal hernia was detected by computed tomography and colonoscopy instead of video capsule endoscopy and upper endoscopy which are usually needed as screening tools for detecting small bowel cancers.

Taken together, the condition that small bowel adenocarcinoma incarcerated within an inguinal hernia was successfully diagnosed preoperatively at stage I and was detected by computed tomography and colonoscopy was extremely rare. The patient neither has clinical symptoms of small bowel adenocarcinoma except for anemia, has family history, nor has predisposing conditions; therefore, preoperative diagnosis of adenocarcinoma of the small bowel was challenging in the current setting $[6,7]$. However, we could detect the tumor preoperatively by performing the colonoscopy for anemia assessment and could perform the definitive cancer surgery and hernia repair. In the case that patients have longstanding incarcerated hernia and anemia, exploration of the terminal ileum by colonoscopy may help to detect small bowel adenocarcinomas which are located in the terminal ileum. By detecting the tumor incarceration within an inguinal hernia in advance, it might be easier to select the appropriate approach for definitive cancer surgery and hernia repair.

\section{Conclusions}

We reported to our knowledge the first case of small bowel adenocarcinoma incarcerated within an inguinal hernia.

\section{Abbreviations \\ $\mathrm{CT}$ : Computed tomography}

Acknowledgements

Not applicable. 


\section{Authors' contributions}

$H K, N T$, and TI performed the operation and supported the postoperative management. KH, MI, and $\mathrm{KY}$ checked and revised the manuscript. All authors read and approved the final manuscript.

\section{Funding}

There is no funding for this work.

\section{Availability of data and materials}

The dataset supporting the conclusion of this article is included within the article.

Ethics approval and consent to participate Not applicable.

\section{Consent for publication}

Written informed consent was obtained from the patient for publication of this report and the use of any accompanying images.

\section{Competing interests}

The authors declare that they have no competing interests.

\section{Author details}

'Department of Surgery, The Saitama Jikei Hospital, 3-208, Ishihara, Kumagaya, Saitama 360-0816, Japan. ${ }^{2}$ Department of Surgery, The Jikei University School of Medicine, 3-25-8, Nishi-Shimbashi, Minato-ku, Tokyo 105-8461, Japan. ${ }^{3}$ Department of Pathology, The Jikei University School of Medicine, 3-25-8, Nishi-Shimbashi, Minato-ku, Tokyo, 105-8461 Japan.

Received: 26 August 2019 Accepted: 20 December 2019

Published online: 27 December 2019

\section{References}

1. Aparicio T, Zaanan A, Svrcek M, Laurent-Puig P, Carrere N, Manfredi S, Locher C, Afchain P. Small bowel adenocarcinoma: epidemiology, risk factors, diagnosis and treatment. Dig Liver Dis. 2014;46:97-104.

2. Halfdanarson TR, McWilliams RR, Donohue JH, Quevedo JF. A singleinstitution experience with 491 cases of small bowel adenocarcinoma. Am J Surg. 2010;199:797-803.

3. Overman MJ, Hu CY, Kopetz S, Abbruzzese JL, Wolff RA, Chang GJ. A population-based comparison of adenocarcinoma of the large and small intestine: insights into a rare disease. Ann Surg Oncol. 2012;19:1439-45.

4. Kanemura T, Takeno A, Tamura S, Okishiro M, Nakahira S, Suzuki R, Nakata K, Egawa C, Miki H, Takeda Y, Kato T. Elective laparoscopic surgery for sigmoid colon carcinoma incarcerated within an inguinal hernia: report of a case. Surg Today. 2014:44:1375-9.

5. Bilimoria KY, Bentrem DJ, Wayne JD, Ko CY, Bennett CL, Talamonti MS. Small bowel cancer in the United States: changes in epidemiology, treatment, and survival over the last 20 years. Ann Surg. 2009;249:63-71.

6. Jess T, Loftus EV Jr, Velayos FS, Harmsen WS, Zinsmeister AR, Smyrk TC, Schleck CD, Tremaine WJ, Melton $\amalg$ 3rd, Munkholm P, Sandborn WJ. Risk of intestinal cancer in inflammatory bowel disease: a population-based study from Olmsted County, Minnesota. Gastroenterology. 2006;130:1039-46.

7. Bernstein CN, Blanchard JF, Kliewer E, Wajda A. Cancer risk in patients with inflammatory bowel disease: a population-based study. Cancer. 2001;91: $854-62$

\section{Publisher's Note}

Springer Nature remains neutral with regard to jurisdictional claims in published maps and institutional affiliations.

\section{Submit your manuscript to a SpringerOpen ${ }^{\circ}$ journal and benefit from:}

- Convenient online submission

- Rigorous peer review

- Open access: articles freely available online

High visibility within the field

- Retaining the copyright to your article 